\title{
THE DYNAMICS OF PLANETARY NEBULAE
}

\author{
BRUCE BALICK \\ Astronomy Dept, University of Washington, \\ P.O. Box 351580, Seattle WA 98195 USA \\ AND \\ ADAM FRANK \\ Dept. of Physics and Astronomy, University of Rochester, \\ Rochester NY 14627-0171 USA
}

\section{Introduction}

In this review we focus on new observations and theory that directly pertain to understanding the hydrodynamic evolution of planetary nebulae (PNe). The recent history of the field lead us to break the review into two parts, macroscopic and microscopic structures, since both the size scales and the dominant physics appear to cleave nicely into more-or-less distinct groups.

Many people are working in this field, and progress is so rapid that similar papers sometimes appear almost simultaneously in the literature. In the interest of space we cannot mention every relevant reference or review any but the most relevant observational papers (see reviews by Manchado, Lopez, and others in this volume). Sometimes we only cite a list of active researchers without mentioning specific papers. We apologize for this. The individual papers will generally appear in more appropriate review and invited papers in this volume.

\section{Observations}

An extensive set of ground based images, spectra, and kinematic observations were already in the literature as of the last IAU symposium (155) on PNe. These ground based observations defined the major classes of macrostructures, of which the fundamental ones are round, elliptical, and bipolar - the shapes that one would see in deep, highly defocussed images. Since then deeper and more accurate image data have proliferated. Sev- 


\section{PN DYNAMICS}

eral subclasses of morphologies have emerged, such as point symmetric (cf several atlases and discussions by Stanghellini, Corradi, Schwarz, Lopez, Manchado, and their many collaborators). Will will not pursue the new subclasses here and simply note that with the exception of point symmetric nebulae they do not change our basic understanding of PN dynamics.

New HST observations have begun to appear (primarily in conference proceedings) over the past three years. These are raising interesting new questions while providing insight into older questions. We shall discuss the HST data - mostly WFPC2 images so far - in more detail below.

\section{Global Dynamics}

The next sections focus on the interpretations of observations in light of new concepts or models that account for the formation and evolution of structure in PNe. The sections are titled by topic. The distinctions are more stylistic than substantive.

The basic conceptual physics is that of the "interacting stellar winds" paradigm originally proposed by Kwok, Purton, and Fitzgerald ${ }^{1}$ in a simple one-dimensional geometry. Since very few PNe are round, the concept had to be extended to two dimensions, and the physics in the models required the inclusion of time-dependent stellar ionization and realistic nebular cooling. The pioneering models were presented at the Innsbruck meeting and published shortly thereafter by Frank and Mellema ${ }^{2,3,4,5}$ and others ${ }^{6,7}$ along with comparisons to observed images and kinematic data.

In order of increasing size, and restricting the discussion to round and elliptical PNe, the basic structural features of PNe which need to be explained are the optically invisible cavity, a thin bright rim (which surrounds the cavity at radius $r_{\text {rim }}$ ), a smooth shell (which surrounds the rim, falls off slowly, and then terminates at radius $\left.r_{\text {shell }}\right)$, and a "detached halo" outside the shell (whose surface brightness tends to increase with radius and then cuts off at an outer radius $r_{\text {halo }}$ ).

The models show that the interaction between fast stellar winds and ambient material form these structures shortly after the fast wind begins and forms a hot bubble whose pressure exceeds that of the confining shell. With respect to the shell the bubble expands supersonically, so the gas shell is swept ahead of it forming a dense, ionized, and bright rim. The basic concepts to account for the morphologies have long been described in the context of "bubble nebulae". Space does not permit a discussion here.

Bipolar nebulae result when the disk-to-pole pressure (i.e. density) contrast is very high. In this case the hot bubble's expansion is extremely fast along the symmetry axis, so the growing hot bubble quickly overtakes the confining shell along the poles. The now-bipolar bubble spills into the low 


\section{B. Balick}

density environment outside at its own sound speed (several hundred $\mathrm{km}$ $\mathrm{s}^{-1}$ ) and cools adiabatically.

In the past four years numerical models have revealed new facets of PN bubble dynamics. In the case of spherical nebulae the interaction of the ionization fronts and the shocks waves has been shown to produce a variety of morphological and kinematic features ${ }^{7,8,9}$. Similar but more compliated features have been found in multi-dimensional simulations of Mellema ${ }^{10}$. Dwarkadas, Chevalier \& Blondin ${ }^{6}$ showed that the initial expansion speed of the shell can influence the development of the nebular shapes both in time scales and in the ranges of shapes that result. Mellema \& Frank ${ }^{4}$ have shown that heating from photoionization can dilute the equator to pole density contrast as the bubbles evolves producing more elliptical bubbles. The effects of sweeping up a weak stellar magnetic field by the stellar winds, and produced a set of analytical models which also conform to observed PN shapes (Chevalier \& Luo ${ }^{11}$, Różyczka, \& Franco ${ }^{12}$, Garcia-Sequra these proceedings). Observational evidence of such fields is unavailable.

A common puzzle emerging from all of these studies is the absolute necessity for the star to form a disk of some sort during the ejection of the shell which we observe today. Without the disk, i.e. the formation of a nondegenerate axis of symmetry, the elliptical and bipolar shapes cannot develop. There is direct evidence that such disks or tori exist in the undisturbed shells. So the real mystery is how the stars form disks.

Mechanisms that can produce precessing ejection and symmetry axes are discussed by M. Livio and others elsewhere in this volume. The most popular of these mechanisms includes tidal effects of a nearby companion, stellar rotation (which flings matter into orbits that intersect in an equatorial plane), and the effects of stellar magnetic fields. All of the models suffer various defects, as Livio describes. Livio and Pringle ${ }^{13}$ argue that the symmetry axis precesses if the disk suffers radiatively induced instabilities.

In all cases, the hot bubble eventually overtakes the shell of old, slow wind. For shells of uniform density, the breaching of the confining shell occurs all along the shell radius at about the same time, and instabilities might result ${ }^{14}$. In the more general case the growing prolate bubble reaches the edge of the confining shell first at the poles. Like a gas in a balloon being pierced at opposite points, the hot bubble emerges quickly at the poles, deflates, and generally forms two lobes of escaping gas around the edges of a barrel-shaped shell remnant.

The outer halos, on the other hand, appear to be the result of a much older mass ejection from the star, perhaps even a direct result of a previous thermal flash in the AGB star ${ }^{15}$. The dilution of the mass in this large structure explains its very low emission measure, and the increase of surface brightness with radius is the result of sweeping up of low-density ambient 


\section{PN DYNAMICS}

gas (i.e. a forward ram pressure as seen by the outflowing material) as well as backflows and projection effects. Hereafter we ignore halos.

\section{Global Instabilities}

Images of PNs show a variety of structure on scales smaller than the rim, shell, halo. In some cases these appear as a network of filaments seemingly seen in projection in the bright rims of $\mathrm{PNe}$, especially when the rims are near the outer edge of the shell. The declining pressures at the edge of the shell presumably lead to the acceleration of the bubble's growth, making conditions suitable for Rayleigh-Taylor (R-T) instabilities along the edge. Kahn \& Breitschwert ${ }^{14}$ studied the process when the shock driven by the expanding ionization front reaches the shell edge. The same process might set in later when the bubble-driven rim reaches the shell's edge ${ }^{16}$.

\section{Microstructures}

Microstructures are very small structures, generally unresolved in groundbased images, and first detected owing to their exctinction or anomalous emergent spectra (they are typically much lower in ionization than the rest of the nebula). Often the microstructures are found in clumps near the outer edges of rims or shells.

New WFPC2 images show very clearly that the morphologies, and perhaps the formation processes of microstructures vary greatly from one PN to the next. Indeed, the only characteristic in common appears to be the smallest size scales, about $10^{15} \mathrm{~cm}$ or so. This scale is also the size of shocks and ionization fronts. It also just happens to match the resolution of the planetary camera, $0 . " 05$, an ominous coincidence. We discuss specific nebulae since there are too few WFPC2 images to draw sweeping conclusions.

Several of the WFPC2 images of NGC 6720, NGC 7207, and IC 4406 presented at this meeting by Bond show hundreds of small dark cloudlets silhouetted against the outer regions of their bright shells. These nebulae also exhibit radio $\mathrm{CO}$ emission from the same general regions. Apparently the formation of such neutral, dense knots is possible during the protoPN evolutionary phase. The formation process is not understood, though a belt of huge comets might explain their masses.

Cometary nebulae eminating from similar dark knots are found near the perimeter of the Helix Nebula ${ }^{17}$. Their pencil-thin extend radially outward from each dark knot. As discussed by O'Dell elsewhere in this volume, photoheated material which evaporates from the leading edge of the knot seems to be caught up in a gentle "breeze" from the central part of the nebula. The neutral knots at the heads of the comets lie in the former constraining waist of an elliptical PN, much as in Bond's nebulae. Seemingly 


\section{B. Balick}

the regions between the knots has been eroded, and remnant hot bubble gas now sweeps past them.

Balick et al. ${ }^{18,19,20}$ have presented WFPC2 images of elliptical PNe with FLIERs at this meeting, some reproductions of which appear elsewhere in this volume. FLIERs, or Fast, Low-Ionization Emission Regions, have long been known to have strangely low ionizations (e.g. they are seen primarily or exclusively in lines such as [NII] and [OI]) and highly supersonic speeds of $30-50 \mathrm{~km} \mathrm{~s}^{-1}$.

Once thought to be N-rich stellar spitballs ${ }^{20}$, the WFPC2 images show that a more likely description is one in which mass-loaded flows are produced behind quiescent knots. FLIERs tend to come in clusters, suggesting that the winds in which they are immersed are collimated and/or the knots were formed in groups along the PN symmetry axis during the protoPN phase $^{21}$.

At the extreme end of dynamically active microstructures are the cometary nebulae in Abell 30 discussed by Borkowski et $\mathrm{al}^{22}$. Like the cometary nebulae of the Helix nebulae, those in A 30 are very thin (with exceptions) and point away from the center of the nebula. However, the knots at the head of the tails are very highly ionized. Meaburn and Lopez ${ }^{23}$ find that the knots have speeds of about $30 \mathrm{~km} \mathrm{~s}^{-1}$ (like FLIERs), and behind the tails the gas motions surpass $100 \mathrm{~km} \mathrm{~s}^{-1}$. Borkowski et al. suggest that the knots are exposed to a combination of photon and collisional heating from a very fast wind in which they are immersed. The evaporating material becomes caught up in the winds, and as it does so the hot winds are cooled and decelerated. The high speeds of the visible gas may be the result of the entrained material in these "mass loaded flows" (Dyson et al. ${ }^{24}$ ).

The processes that form microstructures and associated dense knots have not been identified. One possibility is instabilities that form early in the lifetime of the nebula when the gas is largely neutral, cools efficiently, and its thermal pressures are low. Additionally, microstructures might form as instabilities described by Vishniac ${ }^{25,26}$, though in this case models by Garcia-Segura \& MacLow ${ }^{27}$ show that radial fingers of dense gas interior to the shell will be observable manifestations. Such fingers are not generally observed except, perhaps, in the FLIERs of NGC 7662. (For this nebula, however,the fingers appear at the limb of the shell, suggesting that they formed as R-T instabilities.)

\section{Jets and Ansae}

Some of the most puzzling features of PNe are jets and ansae ("handles") that appear outside the nebular shell. Excellent examples of jets are Hubble 4 and NGC 7354; ansae are found in NGC 6210, NGC 7009, and IC 


\section{PN DYNAMICS}

4593. (Browse the compilation of PN images by Manchado et al. ${ }^{27}$ which became available at this conference.) In both cases highly collimated flows must create and maintain the structures. The same morphologies have been detected in cool protoPNs such as the Frosty Leo Nebula, CRL 2688, and others.

No sustained collimation mechanisms that operate when the nebula is photoionized and the bubble is heated by fast winds had been identified by the last IAU meeting ${ }^{29}$. The local thermal pressures are too high and the cooling processes too inefficient for gas to condense. As pointed out by Frank et al. ${ }^{21}$, collimated flows can, and perhaps are even likely, to form in elliptical PNe during the cold protoPN phase. The important mechanism, converging conical flows, was originally proposed by Canto et al. ${ }^{30}$ for explaining the formation of Herbig-Haro objects in YSOs. Once a disk of dense, cold material forms around an AGB star the same mechanism should operate, at least until the stellar wind speeds exceed about $150 \mathrm{~km}$ $\mathrm{s}^{-1}$ when the cooling processes change their character and conditions for focussing flows lose their impact.

\section{Looking Forward}

The broad goal, as always, is to improve our understanding of the mechanisms that influence the formation and evolution of gaseous systems. $\mathrm{PNe}$ are especially productive astrophysical "subjects" since they are relatively simple, easily observed, and long studied systems which help us to develop an intuition and a set of tools that might be applied to less cooperative but still interesting systems such as YSOs, LBVs, and AGNs.

There is lots to learn.

\section{MACROSTRUCTURES}

What are the mechanisms that lead to microstructure formation?

What are the mechanisms that lead to jet and ansae formation?

What are the mechanisms that lead to point symmetric nebulae formation?

When are each or all of these mechanisms active?

\section{MICROSTRUCTURES}

How do microstructures form and evolve?

Why is their ionization so atypical of their immediate surroundings?

Are many of them heavily enriched in the byproducts of stellar nucleosynthesis, as spectroscopic observations now suggest? Does this help to understand their origins and expulsion mechanism?

Can the microstructures be identified with the mass-loaded flows?

If so, what is the physics of the mass ablation, and how does the heat actually reach their surfaces? 


\section{B. Balick}

\section{References}

Balick B., Peston, H.L., \& Icke V., 1987, AJ, 94, 958

Balick B., Rugers, M., Terzian, Y. \& Chengular, J.N., 1993, ApJ, 411, 778

Balick B., Perinotto, M., Maccioni, A., Terzian, Y., \& Hajian, A., 1994, ApJ, 424, 800

Borkowski, K.J., Kazimierz J., Harrington, J. P, Tsvetanov, Z. I., 1995, ApJ, 449, 143L

Canto J., Tenorio-Tagle G., \& Rozyckza M., 1988, A\&A, 192, 287

Capriotti E.R., 1973, ApJ, 1979, 495

Chevalier, R.A, \& Luo D., 1994, ApJ, 421, 225

Dwarkadas V.V., Chevalier R.A, \& Blondin J.M., 1996, ApJ, 457773

Dyson J.E.; Hartquist T.W.; Biro S., 1993, MNRAS, 261, 430

Frank A., \& Mellema G., 1994a, A\&A, 278, 320

Frank A., \& Mellema G., 1994b, ApJ, 430, 800

Frank A., 1994a, AJ, 107, 256

Frank A., Balick B., \& Livio M., 1996, ApJ, 471, L53

Garcia-Sequra G., \& MacLow M., 1995, ApJ, 455, 160

Hajian A., Frank A., Balicj B., \& Terzian Y., 1996, ApJ, in press

Icke V., Mellema G., Balick B., Eulderink F., Frank A. 1992, Nature, 355, 524.

Kahn F.D., \& Breitschwert, D. 1990, MNRAS 242505

Kwok S., Purton C.R., \& Fitzgerald M.P., 1978, ApJ, 219 125L

Livio M., \& Pringle J.E., 1996, ApJ, 465, 55L

Manchado A., Guerrero M., Stanghellini L., \& Serra-Ricart M., 1996, "The IAC Catalog of Northern Galactic PNe", (IAC, La Laguna)

Marten H., \& Schonenberner D., 1991, A\&A, 248, 590

Meaburn J., \& Lopez J.A., 1996, (preprint)

Mellema G., \& Frank A., 1995a, MNRAS, 273, 401

Mellema G., \& Frank A., 1995b, in "Asymmetrical Planetary Nebulae", Annals of the Isreal Physical Society, ed. N. Soker, A. Harpaz

Mellema G., 1994, A\&A, 290, 915

Mellema G., 1995, MNRAS, 277, 173

O'Dell C.R., \& Handron K.D., 1996 AJ, 111, 1630

Różyczka \& Franco J., 1996, ApJ, 469, 127L

Ryu D., \& Vishniac E.T., 1988, ApJ, 331, 350

Vishniac E.T., 1994, ApJ, 428, 186 